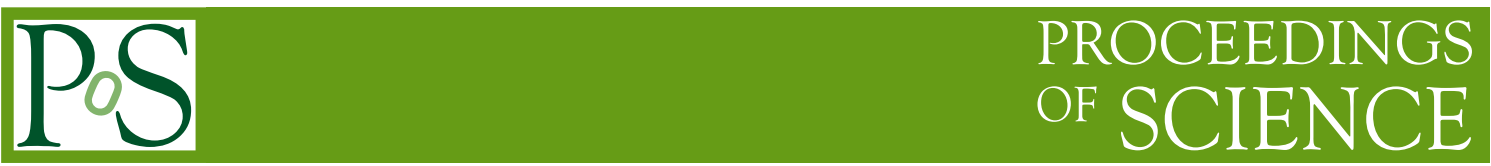

DESY 09-011

SFB-CPP-09/17

\title{
From Moments to Functions in Quantum Chromodynamics
}

\author{
Johannes Blümlein *† \\ Deutsches Elektronen-Synchrotron, DESY, \\ Platanenallee 6, D-15738 Zeuthen, Germany \\ E-mail: Johannes.Bluemlein@desy.de \\ Manuel Kauers \\ Institute for Symbolic Computation (RISC), \\ Johannes Kepler University, Altenberger Straße 69, A-4040 Linz, Austria \\ E-mail: Manuel.Kauers@risc.uni-linz.ac.at

\section{Sebastian Klein} \\ Deutsches Elektronen-Synchrotron, DESY, \\ Platanenallee 6, D-15738 Zeuthen, Germany \\ E-mail: Sebastian.Klein@desy.de

\section{Carsten Schneider} \\ Institute for Symbolic Computation (RISC), \\ Johannes Kepler University, Altenberger Straße 69, A-4040 Linz, Austria \\ E-mail: cschneiderisc.uni-linz.ac.at
}

\begin{abstract}
Single-scale quantities, like the QCD anomalous dimensions and Wilson coefficients, obey difference equations. Therefore their analytic form can be determined from a finite number of moments. We demonstrate this in an explicit calculation by establishing and solving large scale recursions by means of computer algebra for the anomalous dimensions and Wilson coefficients in unpolarized deeply inelastic scattering from their Mellin moments to 3-loop order.
\end{abstract}

XII Advanced Computing and Analysis Techniques in Physics Research

November 3-7 2008

Erice, Italy

\footnotetext{
* Speaker.

$\dagger$ This project was supported in part by German DFG SFB TR-9, Studienstiftung des Deutschen Volkes, projects P19462-N18, P20162-N18, and P20347-N18 of the Austrian FWF, and EC MRTN HEPTOOLS, contract MRTN-CT2006-035505.
} 


\section{Introduction}

Higher order calculations in Quantum Field Theories easily become tedious due to the larger number of terms emerging and the sophisticated form of the contributing Feynman parameter integrals. This applies already to zero scale and single scale quantities. Even more this is the case for problems containing more than one scale. While in the latter case the mathematical structure of the solution of the Feynman integrals is widely unknown, it is explored to a certain extent for zeroand single scale quantities. Zero scale quantities emerge as the expansion coefficients of the running couplings and masses, as fixed moments of splitting functions, etc. They can be expressed by rational numbers and certain special numbers as multiple zeta-values $(M Z V s)[1,2]$ and related quantities.

Single scale quantities depend on a scale $z$ which may be given as a ratio of Lorentz invariants $s^{\prime} / s$ in the respective physical problem. One may perform a Mellin transform over $z$

$$
\int_{0}^{1} d z z^{N-1} f(z)=M[f](N) .
$$

All subsequent calculations are then carried out in Mellin space and one assumes $N \in \mathbf{N}, N>0$. By this transformation the problem at hand becomes discrete. One may seek a description in terms of difference equations. Zero scale problems are obtained from single scale problems treating $N$ as a fixed integer or considering the limit $N \rightarrow \infty$.

A main question concerning zero scale quantities is: Do the corresponding Feynman integrals always lead to MZVs? In the lower orders this is the case. However, starting at some order, even for single-mass problems, other special numbers will occur [3]. This makes it difficult to use methods like PSLQ [4] to determine the analytic structure of the corresponding terms even if one may calculate them numerically at high enough precision since one has to known the respective basis completely. ${ }^{1}$

Zero scale problems are much easier to calculate than single scale problems. In some analogy to the determination of the analytic structure in zero scale problems through integer relations over a known basis (PSLQ) one may think of an automated reconstruction of the all- $N$ relation out of a finite number of Mellin moments given in analytic form. This is possible for recurrent quantities. At least up to 3-loop order, presumably even to higher orders, single scale quantities belong to this class. Here we report on a general algorithm for this purpose, which we applied to the problem being currently the most sophisticated one: the determination of the anomalous dimensions and Wilson coefficients to 3-loop order for unpolarized deeply-inelastic scattering [8]. Details of our calculation are given in Ref. [9].

\section{Single Scale Feynman Integrals as Recurrent Quantities}

For a large variety of massless problems single scale Feynman integrals can be represented as

\footnotetext{
${ }^{1}$ In a recent analysis [2] the relations between all MZVs in the non-alternating and alternating case were determined up to weight $w=12$ and up to $w=24$ in the non-alternating case using shuffle-, stuffle- and generalized doubling relations. These relations lead to basis lengths according to the conjectures by Broadhurst [5] and Broadhurst-KreimerZagier [6], which meets the upper bound set by Terasoma, Goncharov and Deligne [7]. In the non-alternating case we verified that the latter bound is valid at least to $w=26$ and stopped the calculation afterwards due to the large complexity involved. All this does not exclude the existence of exotic relations reducing the basis further.
} 
polynomials in the ring formed of the nested harmonic sums $S_{a_{1}, \ldots, a_{k}}(N),[10,11]$ and the MZVs $\zeta_{a_{1}, \ldots, a_{l}}$ over the rational function field $\mathbf{Q}(N)$. Here,

$$
\begin{aligned}
S_{b, \vec{a}}(N) & =\sum_{k=1}^{N} \frac{(\operatorname{sign}(b))^{k}}{k^{|b|}} S_{\vec{a}}(k), & & a_{i}, b \in \mathbf{Z} \backslash\{0\}, \\
\zeta_{b, \vec{a}} & =\sum_{k=1}^{\infty} \frac{(\operatorname{sign}(b))^{k}}{k^{|b|}} S_{\vec{a}}(k), & & a_{i}, b \in \mathbf{Z} \backslash\{0\} .
\end{aligned}
$$

If $b=1$, the meaning of $\zeta_{b, \vec{a}}$ is symbolic, since it diverges. The degree of divergence is a positive power of $S_{1}(\infty)$. Rational functions in $N$ and harmonic sums obey recurrence relations. Thus, due to closure properties [14] also any polynomial expression in such terms is a solution of a recurrence. Consider as an example the recursion

$$
F(N+1)-F(N)=\frac{\operatorname{sign}(a)^{N+1}}{(N+1)^{|a|}} .
$$

It is solved by $S_{a}(N)$. Corresponding difference equations hold for harmonic sums of deeper nestedness. Feynman integrals can often be decomposed into a combination containing terms of the form

$$
\int_{0}^{1} d z \frac{z^{N-1}-1}{1-z} H_{\vec{a}}(z), \quad \int_{0}^{1} d z \frac{(-z)^{N-1}-1}{1+z} H_{\vec{a}}(z)
$$

with $H_{\vec{a}}(z)$ being a harmonic polylogarithm, [12]. This structure also leads to recurrences, cf. [13]. It is very likely that single scale Feynman diagrams do always obey difference equations.

\section{Establishing and Solving Recurrences}

We assume that a sufficiently large set of moments at integer values $N_{i}$ is given for a physical quantity, which obeys a recurrence relation. One seeks

$$
\sum_{k=0}^{l}\left[\sum_{i=0}^{d} c_{i, k} N^{i}\right] F(N+k)=0 .
$$

The method for determining potential recurrences is available in standard packages [14]. The corresponding linear system is dense. Rational number arithmetics is not feasible for the large systems to be solved. Let us consider as an example the difference equation being associated to the contribution of the color factor $C_{F}^{3}$ for the 3-loop Wilson coefficient $C_{2, q}^{(3)}$ in unpolarized deeply inelastic scattering. $11 \mathrm{~Tb}$ of memory would be required to establish (3.1) in a naive way. Therefore refined methods have to be applied. We use arithmetic in finite fields together with Chinese remaindering [15], which reduces the storage requirements to a few $\mathrm{Gb}$ of memory. The linear system approximately minimizes for $l \approx d$. If one finds more than one recurrence the different recurrences are joined to reduce $l$ to a minimal value. It seems to be a general phenomenon that the recurrence of minimal order is this with the smallest integer coefficients, cf. also [16]. For even larger problems than those dealt with in the present paper, a series of further technical improvements may be carried out, [17]. 
For the solution of the recurrence low orders are clearly preferred. It is solved in depth-optimal $\Pi \Sigma$ fields [18]; here we apply advanced symbolic summation methods as: efficient recurrence solvers and refined telescoping algorithms. They are available in the summation package Sigma [19] implemented in the computer algebra system Mathematica.

The solutions are found as linear combinations of rational terms in $N$ combined with functions, which cannot be further reduced in the $\Pi \Sigma$ fields. In the present application they turn out all to be nested harmonic sums $S_{\vec{b}}(N)$, 2.1). Other or higher order applications may lead to sums of different type as well, which are uniquely found by the present algorithm.

\section{Determination of the 3-Loop Anomalous Dimensions and Wilson Coefficients}

We apply the method to determine the unpolarized anomalous dimensions and Wilson coefficients to 3-loop order. Here we use the above method to all the contributions to a single color/ $\zeta_{i}$-factor. These are 186 terms. As input to the calculation we use the respective Mellin moments, which were calculated by a MAPLE-code based on the harmonic sum representation [8]. We need very high moments and calculate the input recursively. As an example, let us illustrate the size of the moments for the $C_{F}^{3}$-contribution to the Wilson coefficient $C_{2, q}(N)$. The highest moment required is $N=5114$. It cannot be calculated simply with summer [11] because of time and storage reasons. The highest moment is a rational number with a numerator of 13388 and a denominator 13381 digits. Below we give the moments for $N=3$ and $N=500$.

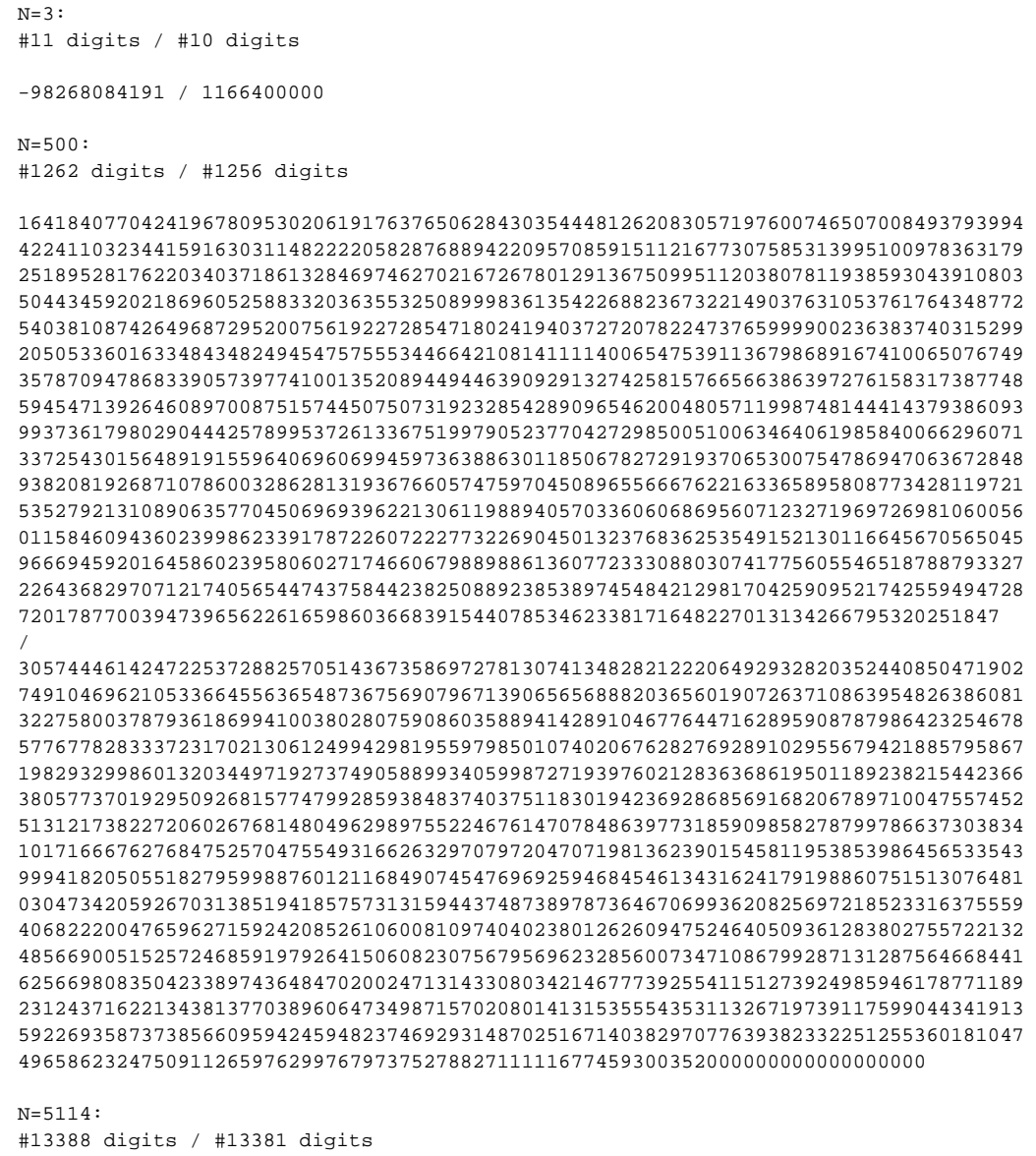


The corresponding difference equations (3.1) are determined by a recurrence finder. Furthermore, the order of the difference equation is reduced to the smallest value possible. The difference equations are then solved order by order using the summation package Sigma.

For the $C_{F}^{3}$-term in $C_{2, q}(N)$ the recurrence was established after 20.7 days of CPU time. Here $4 \mathrm{~h}$ were required for the modular prediction of the dimension of the system, 5.8 days were spent on solving modular linear systems, and 11 days for the modular operator GCDs. The Chinese remainder method and rational reconstruction took 3.8 days. 140 word size primes were needed. As output one obtains a recurrence of $31 \mathrm{Mb}$, which is of order 35 and degree 938, with a largest integer of 1227 digits. The recurrence was solved by Sigma after 5.9 days. We reached a compactification from 289 harmonic sums needed in [8] to 58 harmonic sums, where the representation in [8] (see a corresponding attachment) has already been compactified following an idea of one of the present authors. The determination of the 3-loop anomalous dimensions is a much smaller problem. Here the computation takes about $18 \mathrm{~h}$ only for the complete result.

For the three most complicated cases, establishing and solving of the difference equations took $3+1$ weeks each, requiring $\leq 10 \mathrm{~Gb}$ on a $2 \mathrm{GHz}$ processor. This led to an overall computation time of about sixteen weeks, with the possibility to parallelize four times. Here we did not yet consider parallelization w.r.t. the 140 primes chosen, which would significantly reduce the computational time of the $C_{F}^{3}$ term discussed above and for other comparably large contributions.

In the final representation, we account for algebraic reduction [20]; for this task we used the package HarmonicSums [21] which complements the functionalities of Sigma. One observes that different color factor contributions lead to the same, or nearly the same, amount of sums at a given quantity. This points to the fact that the amount of sums contributing, after the algebraic reduction has been carried out, is governed by topology rather than the field- and color structures being involved. The linear harmonic sum representations used in [8] require many more sums than in the representation reached by the present analysis. A further reduction can be obtained using the structural relations, which leads to maximally 35 different sums up to the level the 3-loop Wilson coefficients [13]. It is not unlikely that the present method can be applied to single scale problems in even higher order. As has been found before in [13,22-24] representing a large number of 2- and 3-loop processes in terms of harmonic sums, the basis elements emerging are always the same. This applies to the anomalous dimensions and Wilson coefficients of the space- and time-like polarized and unpolarized case, the polarized and unpolarized Drell-Yan process and hadronic Higgs-Boson production in the heavy mass limit, deep-inelastic heavy flavor production in the limit $Q^{2} \gg m_{Q}^{2}$, higher order QED corrections in $e^{+} e^{-}$annihilation, as well as to soft and virtual corrections to Bhabha scattering.

In practice no method does yet exist to calculate such a high number of moments ab initio as required for the determination of the all $N$ formulae in the 3-loop case. On the other hand, a proof of existence has been delivered of a quite general and powerful automatic difference-equation solver, standing rather demanding tests, which is ready to help in the solution of present day problems in higher order Quantum Field Theory. It opens up good prospects for the development of even more powerful methods. 


\section{Conclusions}

We established a general algorithm to calculate the exact expression for single scale quantities from a finite (suitably large) number of moments, which are zero scale quantities. The latter ones are much more easily calculable than single scale quantities. We applied the method to the anomalous dimensions and Wilson coefficients up to 3-loop order. To solve 3-loop problems this way is not possible at present, since the number of required moments is too large for the methods available. We established and solved the recurrences for all color resp. $\zeta$-projections at once, which forms a rather voluminous problem. Yet we showed that giant difference equations [order 35; degree $\sim 1000$ ] can be reliably and fast established and solved unconditionally for the most advanced problems in Quantum Field Theory.

Acknowledgment. We would like to thank J. Vermaseren for discussions.

\section{References}

[1] J. M. Borwein, D. M. Bradley, D. J. Broadhurst and P. Lisonek, Trans. Am. Math. Soc. 353 (2001) 907 [arXiv:math/9910045].

[2] J. Blümlein, D. Broadhurst, J. Vermaseren, The multiple zeta value data mine, DESY 09-003.

[3] D. J. Broadhurst, Eur. Phys. J. C 8 (1999) 311 [arXiv:hep-th/9803091];

F. Brown, arxiv:0804.1660 [math.AG];

Y. Andre, Proceedings of the International Conference "Motives, Quantum Field Theory, an Pseudo Differential Operators", Clay Mathematical Institute, Boston, June, 2008.

[4] H.R.P. Ferguson and D.H. Bailey, D. H. A Polynomial Time, Numerically Stable Integer Relation Algorithm, RNR Techn. Rept. RNR-91-032, Jul. 14, 199.

[5] D. J. Broadhurst, arXiv:hep-th/9604128.

[6] D. J. Broadhurst and D. Kreimer, Phys. Lett. B 393 (1997) 403, [arXiv:hep-th/9609128]; D. Zagier, in : First European Congress of Mathematics, Vol. II, (Paris, 1992), Progr. Math., 120, (Birkhäuser, Basel-Boston, 1994), pp. 497.

[7] A.B. Goncharov, arxiv:math.AG/0103059;

T. Terasoma, Invent. Math. 149 (2) (2002) 339, arxiv:math.AG/010423;

P. Deligne and A.B. Goncharov, Ann. Sci. Ecole Norm. Sup., Série IV 38 (1) (2005) 1.

[8] S. Moch, J. A. M. Vermaseren and A. Vogt, Nucl. Phys. B 688 (2004) 101 [arXiv:hep-ph/0403192]; Nucl. Phys. B 691 (2004) 129 [arXiv:hep-ph/0404111]; Nucl. Phys. B 724 (2005) 3 [arXiv:hep-ph/0504242].

[9] J. Blümlein, M. Kauers, S. Klein, and C. Schneider, arxiv: 0902.4091 [hep-ph].

[10] J. Blümlein and S. Kurth, Phys. Rev. D 60 (1999) 014018 [arXiv:hep-ph/9810241];

[11] J. A. M. Vermaseren, Int. J. Mod. Phys. A 14 (1999) 2037 [arXiv:hep-ph/9806280].

[12] E. Remiddi and J. A. M. Vermaseren, Int. J. Mod. Phys. A 15 (2000) 725-754, [arXiv:hep-ph/9905237].

[13] J. Blümlein, Nucl. Phys. Proc. Suppl. 183 (2008) 232 [arXiv:0807.0700 [math-ph]]; arxiv:0901.0837, arxiv:0901.3106. 
[14] B. Salvy and P. Zimmermann, ACM Transactions on Mathematical Software, 20 (2) (1994) 163; C. Mallinger, Master Thesis, J. Kepler University, Linz, (1996).

[15] K.O. Geddes, S. R. Czapor and G. Labahn, Algorithms for Computer Algebra, (Kluwer, Dordrecht, 1992);

J. von zur Gathen and J. Gerhard, Modern Computer Algebra, (Cambridge University Press, Cambridge, 1999);

M. Kauers, Nucl. Phys. B (Proc. Suppl.) 183 (2008) 245;

[16] A. Bostan and M. Kauers, The full counting function for Gessel walks is algebraic, INRIA-Rocquencourt report, 2009, in preparation.

[17] B. Beckermann and G. Labahn, Numerical Algorithms, 3 (1992) 45; SIAM Journal of Matrix Analysis and Applications, 22 (1) (2000) 114.

[18] M. Karr, J. ACM, 28 (1981) 305; J. Symbolic Comput., 1 (1985) 303;

C. Schneider, PhD thesis, RISC-Linz, J. Kepler University, Linz, May 2001; J. Differ. Equations Appl., 11(9) (2005) 799; J. Algebra Appl., 6 (3) (2007) 415; Symbolic summation finds optimal nested sum representations SFB-Report 2007-26, SFB F013, J. Kepler University Linz, 2007; Parameterized telescoping proves algebraic independence of sums Ann. Comb., to appear, 2008; J. Symbolic Comput., 43 (9) (2008) 611.

[19] C. Schneider, Sém. Lothar. Combin., 56 (2007) 1, Article B56b.

[20] J. Blümlein, Comput. Phys. Commun. 159 (2004) 19 [arXiv:hep-ph/0311046].

[21] J. Ablinger. Diploma thesis, J. Kepler University Linz, 2009.

[22] J. Blümlein and V. Ravindran, Nucl. Phys. B 716 (2005) 128 [arXiv:hep-ph/0501178]; Nucl. Phys. B 749 (2006) 1 [arXiv:hep-ph/0604019];

J. Blümlein and S. Moch, in preparation;

J. Blümlein and S. Klein, arXiv:0706.2426 [hep-ph].

[23] J. Blümlein, A. De Freitas, W. L. van Neerven and S. Klein, Nucl. Phys. B 755 (2006) 272 [arXiv:hep-ph/0608024];

M. Buza, Y. Matiounine, J. Smith, R. Migneron and W. L. van Neerven, Nucl. Phys. B 472 (1996) 611 [arXiv:hep-ph/9601302];

I. Bierenbaum, J. Blümlein, S. Klein and C. Schneider, Nucl. Phys. B 803 (2008) 1 [arXiv:0803.0273 [hep-ph]];

I. Bierenbaum, J. Blümlein and S. Klein, Nucl. Phys. B 780 (2007) 40 [arXiv:hep-ph/0703285]; arxiv: 0901.0669, Phys. Lett. B (2009) in print.

[24] F. A. Berends, W. L. van Neerven and G. J. H. Burgers, Nucl. Phys. B 297 (1988) 429 [Erratum-ibid. B 304 (1988) 921];

J. Blümlein, A. De Freitas, W. van Neerven, PoS RADCOR2007 (2007) 005 [arXiv:0812.1588 [hep-ph]]. 